\title{
EXTERIOR PROBLEM OF THE DARWIN MODEL AND ITS NUMERICAL COMPUTATION
}

\author{
LUNG-AN YING ${ }^{1}$ AND FENGYAN LI ${ }^{2}$
}

\begin{abstract}
In this paper, we study the exterior boundary value problems of the Darwin model to the Maxwell's equations. The variational formulation is established and the existence and uniqueness is proved. We use the infinite element method to solve the problem, only a small amount of computational work is needed. Numerical examples are given as well as a proof of convergence.
\end{abstract}

Mathematics Subject Classification. 35Q60, 65N30, 35J50.

Received: May 9, 2002. Revised: March 21, 2003.

\section{INTRODUCTION}

The Darwin model is an approximation model $[5,7]$ for the Maxwell's equations:

$$
\begin{aligned}
\frac{1}{c^{2}} \frac{\partial E}{\partial t}-\nabla \times B & =-\mu_{0} J \\
\frac{\partial B}{\partial t}+\nabla \times E & =0, \\
\nabla \cdot E & =\frac{1}{\varepsilon_{0}} \rho, \\
\nabla \cdot B & =0 .
\end{aligned}
$$

where $E, B$ are the electric field and the magnetic flux density respectively, and $\rho, J$ are the charge and current densities, satisfying

$$
\frac{\partial \rho}{\partial t}+\nabla \cdot J=0
$$

The positive constants $c, \varepsilon_{0}, \mu_{0}$ are the light velocity, the electric permittivity, and the magnetic permeability of vacuum respectively.

There have been some work done on Darwin model. It was shown in [2] that the Darwin model approximates the Maxwell equations up to the second order for $B$ and to the third order for $E$, provided $\eta=\bar{v} / c$ is small, where $\bar{v}$ is a characteristic velocity. In [1] variational formulation and the finite element method for the Darwin

Keywords and phrases. Darwin model, Maxwell's equations, exterior problem, infinite element method.

1 School of Mathematical Sciences, Peking University, Beijing 100871, PR China. e-mail: yingla@math.pku.edu.cn

2 School of Mathematical Sciences, Peking University, PR China.

Current adress: Division of Applied Math., Brown University, RI 02912, USA. e-mail: fengyan-1@cfm.brown.edu

(c) EDP Sciences, SMAI 2004 
model was studied. Two kinds of variational formulation were given. Well posedness and error estimates were proved.

To the authors' knowledge, works in the literature on this model are limited to the bounded domain cases. However, quite a number of problems in application are related to the unbounded domain, and this is why we come to this paper.

Let us recall the model for bounded domain first. Suppose we have an open, bounded, simply connected domain $\Omega$ in $\mathbb{R}^{n}(n=2$ or 3$)$. $\Gamma_{i}, 0 \leq i \leq m$, are the connected components of the boundary $\partial \Omega$ with the unit outward normal $\nu$, and $\Gamma_{0}$ is the outer boundary. Notice that the electric field $E$ can be written as the sum of a transverse component $E_{T}$ and a longitudinal component $E_{L}$, that is,

$$
E=E_{T}+E_{L}
$$

and

$$
\nabla \cdot E_{T}=0, \quad \nabla \times E_{L}=0
$$

Then equation (1) becomes

$$
\nabla \times B=\mu_{0} J+\frac{1}{c^{2}} \frac{\partial E_{L}}{\partial t}+\frac{1}{c^{2}} \frac{\partial E_{T}}{\partial t} .
$$

In order to get the Darwin Model, we neglect the last term in above equation and get

$$
\nabla \times B=\mu_{0} J+\frac{1}{c^{2}} \frac{\partial E_{L}}{\partial t}
$$

Then the system (2)-(4), (7) with initial and boundary conditions

$$
\begin{gathered}
E \times\left.\nu\right|_{\partial \Omega}=0,\left.\quad \frac{\partial}{\partial t} B \cdot \nu\right|_{\partial \Omega}=0 \\
\left.E\right|_{t=0}=E_{0},\left.\quad B\right|_{t=0}=B_{0}
\end{gathered}
$$

will be reduced to

(i) $E_{L}=-\nabla \phi$, and $\phi$ satisfies

$$
\begin{aligned}
-\triangle \phi & =\frac{1}{\varepsilon_{0}} \rho \\
\left.\phi\right|_{\Gamma_{i}} & =\alpha_{i}, \quad 0 \leq i \leq m,
\end{aligned}
$$

$\alpha=\left\{\alpha_{i}\right\}$ satisfies

$$
\begin{aligned}
c \frac{\mathrm{d} \alpha}{\mathrm{d} t} & =\frac{1}{\varepsilon_{0}} \int_{\Omega} J \cdot \nabla \chi \mathrm{d} x, \\
\alpha(0) & =\alpha_{0}
\end{aligned}
$$

where $\alpha_{0}$ depends on $E_{0}, c=\left\{c_{i j}\right\}$ is the capacitance matrix. Here $c_{i j}=\left\langle\frac{\partial \chi_{i}}{\partial \nu}, 1\right\rangle_{\Gamma_{j}}$, and $\chi=\left\{\chi_{i}\right\}$ is the solution of

$$
\begin{aligned}
\triangle \chi_{i} & =0, \\
\left.\chi_{i}\right|_{\Gamma_{j}} & =\delta_{i j} .
\end{aligned}
$$


(ii) $B$ satisfies

$$
\begin{aligned}
-\triangle B & =\mu_{0} \nabla \times J, \\
\nabla \cdot B & =0, \\
\left.B \cdot \nu\right|_{\partial \Omega} & =\left.B_{0} \cdot \nu\right|_{\partial \Omega}, \\
(\nabla \times B) \times\left.\nu\right|_{\partial \Omega} & =\mu_{0} J \times\left.\nu\right|_{\partial \Omega} .
\end{aligned}
$$

(iii) $E_{T}$ satisfies

$$
\begin{aligned}
\triangle E_{T} & =\frac{\partial}{\partial t} \nabla \times B, \\
\nabla \cdot E_{T} & =0 \\
E_{T} \times\left.\nu\right|_{\partial \Omega} & =0, \\
\left\langle E_{T} \cdot \nu, 1\right\rangle_{\Gamma_{i}} & =0, \quad 1 \leq i \leq m .
\end{aligned}
$$

The above problems are well posed (see [1]).

Now we can turn to the exterior problem. Let $\partial \Omega$ be a simply closed curve in $\mathbb{R}^{2}$, and $\Omega$ the exterior domain of it. Then we study problem (iii) as an example, the method to problem (ii) is similar. In the following sections, we are going to show that there are nonzero functions $v$ on $\Omega$ which satisfy

$$
\nabla \cdot v=0, \quad \nabla \times v=0, \quad v \times\left.\nu\right|_{\partial \Omega}=0, \quad\langle v \cdot \nu, 1\rangle_{\partial \Omega}=0
$$

Therefore the solutions to (iii) are not unique. It turns out that these solutions will be unique up to a function which is from a two dimensional space and satisfies (16). After introducing a quotient space, we are able to establish the variational formulation for (12)-(15) as well as the numerical method to solve it, namely, we will use the infinite element method, which has been successfully applied to a large class of problems (see [10]). In particular, this method is effective to solve the exterior problems of the Stokes equation [9], the result there is very useful in our algorithm.

The paper is organized as follows. In Section 2, some notations are introduced. Since the result from Stokes equation is used here, in Section 3, we will recall the variational formulation for the Stokes equation in bounded as well as in exterior domains, in particular, a weighted space is introduced for exterior problem. In Section 4, the Darwin model in bounded and exterior domain will be discussed. The infinite element method for the Darwin model is discussed in Section 5 and a convergence result under a semi-norm is obtained. The algorithm of our scheme in Section 6 is followed by some numerical examples in Section 7.

\section{Notations}

From now on, let $\Omega$ be an open domain in $\mathbb{R}^{2}$ with Lipschitz-continuous boundary $\partial \Omega$, it will be either a bounded domain or an exterior domain depending on the problem we are considering. When we come to the exterior problem, we further assume that $\partial \Omega$ is simply closed. The unit outward normal to $\partial \Omega$ is denoted by $\nu$. Furthermore, let $x=\left(x_{1}, x_{2}\right)$ be a typical point in $\mathbb{R}^{2}$.

$L^{2}(\Omega), H^{1}(\Omega), H_{0}^{1}(\Omega), H^{1 / 2}(\partial \Omega)$ (as well as the vector form $\left(L^{2}(\Omega)\right)^{2},\left(H^{1}(\Omega)\right)^{2},\left(H_{0}^{1}(\Omega)\right)^{2},\left(H^{1 / 2}(\partial \Omega)\right)^{2}$, for simplicity, we will just use scalar notation if there is no confusion) are the conventional notations of the Sobolev spaces (see [3], for example). $(\cdot, \cdot),\|\cdot\|_{0}$ are the inner product and norm in $L^{2}(\Omega) \cdot\|\cdot\|_{1},\left.|\cdot|\right|_{1}$ are the norm and seminorm for $H^{1}(\Omega)$ (also for $H_{0}^{1}(\Omega)$ ). We will always denote by $C$ a generic constant.

Moreover, the following Hilbert spaces will be used for bounded domain $\Omega$ :

$$
L_{0}^{2}(\Omega)=\left\{p \in L^{2}(\Omega) ; \int_{\Omega} p \mathrm{~d} x=0\right\},
$$


and

provided with the norm

$$
H(\operatorname{curl}, \operatorname{div} ; \Omega)=\left\{v \in L^{2}(\Omega) ; \nabla \cdot v, \nabla \times v \in L^{2}(\Omega)\right\}
$$

$$
\|v\|_{0, \mathrm{curl}, \mathrm{div}}=\left(\|v\|_{0}^{2}+\|\nabla \cdot v\|_{0}^{2}+\|\nabla \times v\|_{0}^{2}\right)^{\frac{1}{2}} .
$$

When we come to the exterior problem, the following weighted Sobolev space will be very useful (we might assume the origin $o$ is in the interior of $\partial \Omega$ for notation simplicity):

$$
H^{1, *}(\Omega)=\left\{u \in \mathcal{D}^{\prime} ; \nabla u, \frac{u}{|x| \ln |x|} \in L^{2}(\Omega)\right\}
$$

which is a Hilbert space [6], provided with the following norm

$$
\|u\|_{1, *}=\left(\int_{\Omega}|\nabla u|^{2}+\frac{u^{2}}{|x|^{2} \ln ^{2}|x|} \mathrm{d} x\right)^{\frac{1}{2}}
$$

where $\mathcal{D}^{\prime}$ is the distribution space. Actually $H^{1, *}(\Omega)$ is the closure of $C_{0}^{\infty}(\bar{\Omega})$ with respect to the norm $\|\cdot\|_{1, *}$. Likely, denote $H_{0}^{1, *}(\Omega)$ as the closure of of $C_{0}^{\infty}(\Omega)$ under the same norm. It is proved [6] that in $H_{0}^{1, *}(\Omega)$, the semi-norm $|\cdot|_{1}$ is equivalent to $\|\cdot\|_{1, *}$.

\section{Stokes Equation}

\subsection{The bounded domain problem}

The Stokes problem is: find $u, p$, such that

$$
\begin{aligned}
-\triangle u-\nabla p & =f, \quad x \in \Omega, \\
\nabla \cdot u & =0, \quad x \in \Omega, \\
\left.u\right|_{\partial \Omega} & =g,
\end{aligned}
$$

with

$$
\int_{\partial \Omega} g \cdot \nu \mathrm{d} s=0
$$

For functions $g \in H^{1 / 2}(\partial \Omega)$ satisfying $(20)$, a function $u_{0} \in H^{1}(\Omega)$ exists such that $\nabla \cdot u_{0}=0$, and $\left.u_{0}\right|_{\partial \Omega}=g$ (see [8] Lem. 2.4 on p. 22). Let $u^{\prime}=u-u_{0}$ be the new unknown function, then the problem is reduced to a homogeneous one with new right hand side term $f+\triangle u_{0}$. So here only the homogeneous problem is considered.

Define bilinear forms on $H_{0}^{1}(\Omega) \times H_{0}^{1}(\Omega)$ and $H_{0}^{1}(\Omega) \times L_{0}^{2}(\Omega)$,

$$
\begin{aligned}
& a(u, v)=\int_{\Omega} \nabla u \cdot \nabla v \mathrm{~d} x, \\
& b(u, p)=\int_{\Omega}(\nabla \cdot u) p \mathrm{~d} x,
\end{aligned}
$$

then the variational formulation of the Stokes problem is: given a function $f \in H^{-1}(\Omega)$, find $u \in H_{0}^{1}(\Omega)$, $p \in L_{0}^{2}(\Omega)$, such that

$$
\begin{aligned}
a(u, v)+b(v, p) & =\langle f, v\rangle, & & \forall v \in H_{0}^{1}(\Omega), \\
b(u, q) & =0, & & \forall q \in L_{0}^{2}(\Omega) .
\end{aligned}
$$

where $\langle f, v\rangle$ is the duality product between $H^{-1}(\Omega)$ and $H_{0}^{1}(\Omega)$.

The problem admits a unique solution (see $[8]$ ). 


\subsection{The exterior problem}

The same problem (17)-(20) is considered on exterior domain $\Omega$. Generally, the solutions to the exterior Stokes equation do not vanish at the infinity, so the weighted Sobolev spaces $H^{1, *}(\Omega)$ (and $H_{0}^{1, *}(\Omega)$ ) are much suitable functional setting instead of $H^{1}(\Omega)$ (and $H_{0}^{1}(\Omega)$ ).

Again, for $g \in H^{1 / 2}(\partial \Omega)$ satisfying (20), a function $u_{0} \in H^{1}(\Omega)$ with compact support exists such that $\nabla \cdot u_{0}=0,\left.u_{0}\right|_{\partial \Omega}=g$ (see Lem. 3.1 in [4]), so we only need to consider the homogeneous problem.

Hence, the variational formulation for the homogeneous exterior Stokes problem is: given a function $f \in$ $\left(H_{0}^{1, *}(\Omega)\right)^{\prime}$, find $u \in H_{0}^{1, *}(\Omega), p \in L^{2}(\Omega)$, such that

$$
\begin{aligned}
a(u, v)+b(v, p) & =\langle f, v\rangle, & & \forall v \in H_{0}^{1, *}(\Omega), \\
b(u, q) & =0, & & \forall q \in L^{2}(\Omega)
\end{aligned}
$$

where $a(u, v), b(u, q)$ have the same forms as $(21,22)$, and $\langle f, v\rangle$ is the duality product between $\left(H_{0}^{1, *}(\Omega)\right)^{\prime}$ and $H_{0}^{1, *}(\Omega)$.

This problem admits a unique solution [4].

\section{DARWIN MODEL}

\subsection{The bounded domain problem}

First introduce a subset of $H(\operatorname{curl}, \operatorname{div} ; \Omega)$ :

$$
H_{0 c}(\Omega)=\left\{v \in H(\operatorname{curl}, \operatorname{div} ; \Omega) ; v \times\left.\nu\right|_{\partial \Omega}=0\right\},
$$

and also two bilinear forms defined on $H_{0 c}(\Omega) \times H_{0 c}(\Omega)$ and $H_{0 c}(\Omega) \times L^{2}(\Omega)$,

$$
\begin{gathered}
d(u, v)=\int_{\Omega}(\nabla \times u) \cdot(\nabla \times v) \mathrm{d} x+\int_{\Omega}(\nabla \cdot u)(\nabla \cdot v) \mathrm{d} x+\langle u \cdot \nu, 1\rangle_{\partial \Omega}\langle v \cdot \nu, 1\rangle_{\partial \Omega} \\
b(v, p)=\int_{\Omega}(\nabla \cdot v) p \mathrm{~d} x .
\end{gathered}
$$

The following result is proved in [1]

Theorem 4.1. The problem: find $u \in H_{0 c}(\Omega), p \in L^{2}(\Omega)$, such that

$$
\begin{aligned}
d(u, v)+b(v, p) & =\int_{\Omega} B \cdot(\nabla \times v) \mathrm{d} x, \quad \forall v \in H_{0 c}(\Omega), \\
b(u, q) & =0, \quad \forall q \in L^{2}(\Omega),
\end{aligned}
$$

admits a unique solution, and $p \equiv 0$. Moreover, the norm defined by $\sqrt{d(\cdot, \cdot)}$ is equivalent to $\|\cdot\|_{0, \text { curl,div }}$ in $H_{0 c}(\Omega)$.

Remark 4.1. The following observation will be useful for our later discussion: The space $L^{2}(\Omega)$ in the above formulation can be replaced by $L_{0}^{2}(\Omega)$, then the formulation becomes: find $u \in H_{0 c}(\Omega), p \in L_{0}^{2}(\Omega)$, such that

$$
\begin{aligned}
d(u, v)+b(v, p) & =\int_{\Omega} B \cdot(\nabla \times v) \mathrm{d} x, \quad \forall v \in H_{0 c}(\Omega), \\
b(u, q) & =0, \quad \forall q \in L_{0}^{2}(\Omega) .
\end{aligned}
$$

This is because the solution $(u, p)$ to $(29,30)$ satisfies $p \equiv 0$, hence it is also a solution to $(31,32)$. On the other hand, it is easy to prove $(31,32)$ also admits a unique solution. 
Remark 4.2. Notice that the solution to $(29,30)$ satisfies

$$
\langle u \cdot \nu, 1\rangle_{\partial \Omega}=0 .
$$

This comes from the Green formula and the fact $\nabla \cdot u=0$.

\subsection{The exterior problem}

In order to figure out the suitable functional setting for the exterior Darwin problem, we first take a cut-off function $\zeta \in C^{\infty}(\Omega)$, which satisfies: $\zeta \equiv 1$ near the boundary $\partial \Omega, \zeta \equiv 0$ near the infinity, and $0 \leq \zeta \leq 1$. Let $\Omega^{\prime}$ be a bounded subset of $\Omega$ such that $\operatorname{supp}(\zeta) \subset \Omega^{\prime}$. Define

$$
H_{0 c}(\Omega)=\left\{v \in \mathcal{D}^{\prime} ;\|\zeta v\|_{0, \text { curl, div }}<\infty,(1-\zeta) v \in H_{0}^{1, *}(\Omega), v \times\left.\nu\right|_{\partial \Omega}=0\right\},
$$

and

$$
\|u\|_{*}=\left\{\|\nabla \times u\|_{0}^{2}+\|\nabla \cdot u\|_{0}^{2}+\langle u \cdot \nu, 1\rangle_{\partial \Omega}^{2}\right\}^{\frac{1}{2}} .
$$

However, $\|\cdot\|_{*}$ does not provide a norm in $H_{0 c}(\Omega)$, actually we have the following result:

Lemma 4.1. Let $V_{0}=\left\{u \in H_{0 c}(\Omega) ;\|u\|_{*}=0\right\}$, then

$$
V_{0}=\left\{u=\left(u_{1}, u_{2}\right) ; u_{1}=\frac{\partial \phi}{\partial x_{2}}, u_{2}=-\frac{\partial \phi}{\partial x_{1}}, \phi=a\left(x_{2}+f(x)\right)+b\left(x_{1}+g(x)\right),(a, b) \in \mathbb{R}^{2}\right\},
$$

where $f, g \in H^{1, *}(\Omega)$ satisfy

$$
\begin{aligned}
& -\triangle \psi=0, \quad x \in \Omega \\
& \left.\frac{\partial \psi}{\partial \nu}\right|_{\partial \Omega}=-\left.\frac{\partial \chi}{\partial \nu}\right|_{\partial \Omega}
\end{aligned}
$$

when $\chi\left(x_{1}, x_{2}\right)=x_{2}, x_{1}$ separately.

Proof. We take any $u \in V_{0}$, it will satisfy

$$
\begin{aligned}
\nabla \cdot u & =0, \\
\nabla \times u & =0, \\
u \times\left.\nu\right|_{\partial \Omega} & =0, \\
\langle u \cdot \nu, 1\rangle_{\partial \Omega} & =0 .
\end{aligned}
$$

From (33)-(36), $\int_{\Gamma}-u_{2} \mathrm{~d} x_{1}+u_{1} \mathrm{~d} x_{2}=0$ is true for any closed curve $\Gamma$ in $\Omega$. Thus we can define

$$
\phi(x)=\int_{x_{0}}^{x}-u_{2} \mathrm{~d} x_{1}+u_{1} \mathrm{~d} x_{2},
$$

where $x_{0}$ can be any but fixed point in $\Omega$. Notice now $u_{1}=\frac{\partial \phi}{\partial x_{2}}, u_{2}=-\frac{\partial \phi}{\partial x_{1}}$.

From $(34,35)$, we can get

$$
-\triangle \phi=\nabla \times\left(\frac{\partial \phi}{\partial x_{2}},-\frac{\partial \phi}{\partial x_{1}}\right)=\nabla \times u=0,
$$

and

$$
\left.\frac{\partial \phi}{\partial \nu}\right|_{\partial \Omega}=\left(\frac{\partial \phi}{\partial x_{2}},-\frac{\partial \phi}{\partial x_{1}}\right) \times\left.\nu\right|_{\partial \Omega}=u \times\left.\nu\right|_{\partial \Omega}=0 .
$$


Thus starting from every $u \in V_{0}$, we will end up with a problem: find a function $\phi$, satisfying

$$
\begin{aligned}
& -\triangle \phi=0, \quad x \in \Omega \\
& \left.\frac{\partial \phi}{\partial \nu}\right|_{\partial \Omega}=0 .
\end{aligned}
$$

Besides, $u$ is a bounded harmonic function, which can be developed in a neighborhood of the infinity as follows:

$$
u_{1}-i u_{2}=\sum_{k=0}^{\infty} \frac{z_{0 k}}{z^{k}}
$$

where $z_{0 k}=a_{k}+i b_{k},\left(a_{k}, b_{k}\right) \in \mathbb{R}^{2}, z=x+i y=r e^{i \theta}$. That is

$$
\left(u_{1}, u_{2}\right)=\left(\sum_{k=0}^{\infty} \frac{a_{k} \cos k \theta+b_{k} \sin k \theta}{r^{k}}, \sum_{k=0}^{\infty} \frac{a_{k} \sin k \theta-b_{k} \cos k \theta}{r^{k}}\right) .
$$

From (33)-(36) we know $a_{1}=0$, so

$$
u_{1}-i u_{2}=\left(a_{0}+\frac{b_{1} \sin \theta}{r},-b_{0}-\frac{b_{1} \cos \theta}{r}\right)+\sum_{k=2}^{\infty} \frac{z_{0 k}}{z^{k}}
$$

Hence the asymptotic expansion of $\phi(x)$ near the infinity will be

$$
\phi(x) \rightarrow a_{0}\left(x_{2}-x_{20}\right)+b_{0}\left(x_{1}-x_{10}\right)+b_{1}\left(\ln r-\ln r_{0}\right)+O\left(\frac{1}{r}\right)=h(x)+c+O\left(\frac{1}{r}\right),
$$

where $h(x)=a_{0} x_{2}+b_{0} x_{1}+b_{1} \ln r$.

Introduce a new function $\psi$, such that $\phi=\psi+h$. Then $\psi$ will satisfy

$$
\begin{aligned}
& -\triangle \psi=0, \quad x \in \Omega \\
& \left.\frac{\partial \psi}{\partial \nu}\right|_{\partial \Omega}=-\left.\frac{\partial h}{\partial \nu}\right|_{\partial \Omega},
\end{aligned}
$$

Notice that it suffices to consider the solution only in $H^{1, *}(\Omega) / \mathbb{R}$ now.

Knowing that the well posedness of problem (38) is equivalent to

$$
\int_{\partial \Omega} \frac{\partial h}{\partial \nu} \mathrm{d} s=0
$$

so let $D(r)=\{x ;|x|<r\}$, and $\Omega_{r}=\Omega \cap D(r)$, then by the Green formula we get

$$
\int_{\partial \Omega} \frac{\partial h}{\partial \nu} \mathrm{d} s=\int_{\Omega_{r}} \triangle h \mathrm{~d} x-\int_{\partial D(r)} \frac{\partial h}{\partial \nu} \mathrm{d} s=-2 \pi b_{1} .
$$

Therefore $b_{1}=0$ is sufficient and necessary in order that (38) admits a unique solution in $H^{1, *}(\Omega) / \mathbb{R}$.

Once $b_{1}=0$, there exist $f, g \in H^{1, *}(\Omega) / \mathbb{R}$, such that

$$
\psi=a_{0} f(x)+b_{0} g(x)
$$


where $f, g$ are the solutions to (38) with $h(x)=x_{2}, x_{1}$ respectively. Consequently

$$
\phi(x)=a_{0}\left(x_{2}+f(x)\right)+b_{0}\left(x_{1}+g(x)\right) .
$$

On the other hand, it is easy to see that the $u$ given in the Lemma for all $a, b$ are in $V_{0}$.

Let $V=\left\{v \in H_{0 c}(\Omega) ;\langle v \cdot \nu, 1\rangle_{\partial \Omega}=0\right\}$, and let the closure of the quotient space $V / V_{0}$ with respect to the norm $\|\cdot\|_{*}$ be $W$. Besides, let $\mathcal{Q}=\left\{p \in L^{2}(\Omega) ; \operatorname{supp} p \subset \subset \Omega\right\}$, equipped with norm

$$
\|p\|_{\sharp}=\left(\|p\|_{0}^{2}+\left|\int_{\Omega} p \mathrm{~d} x\right|^{2}\right)^{\frac{1}{2}}
$$

Then we take closure to obtain a Hilbert space $Q$. If $p \in Q$ and $\lim p_{n}=p, p_{n} \in \mathcal{Q}$, then we define $\int_{\Omega} p \mathrm{~d} x=$ $\lim \int_{\Omega} p_{n} \mathrm{~d} x$. Notice this can be seen as a generalized integral. The subspace of $Q,\left\{p \in Q ; \int_{\Omega} p \mathrm{~d} x=0\right\}$ is denoted by $Q_{0}$.

Now the variational formulation for the exterior problem will be: find $u \in W, p \in Q_{0}$, such that

$$
\begin{aligned}
d(u, v)+b(v, p) & =\int_{\Omega} B \cdot(\nabla \times v) \mathrm{d} x, \quad \forall v \in W, \\
b(u, q) & =0, \quad \forall q \in Q_{0},
\end{aligned}
$$

here, bilinear forms $d(\cdot, \cdot), b(\cdot, \cdot)$ have the same forms as $(27,28)$.

In order to prove that there is a unique solution to the problem $(39,40)$, we need an auxiliary lemma.

Lemma 4.2. For a given $q \in Q_{0}$ there is a $\left(v_{q}+V_{0}\right) \in W$ such that

$$
\nabla \cdot v_{q}=q, \quad \nabla \times v_{q}=0, \quad v_{q} \times\left.\nu\right|_{\partial \Omega}=0,
$$

consequently

$$
\left\|v_{q}\right\|_{*}^{2}=\|q\|_{0}^{2}=\|q\|_{\sharp}^{2} .
$$

Proof. For a given $\varepsilon>0$, there exists $q_{\varepsilon} \in \mathcal{Q}$ so that $\left\|q-q_{\varepsilon}\right\|_{\sharp}<\varepsilon$. Then consider the following problem: Find $\phi \in H_{0}^{1, *}(\Omega)$, such that

$$
(\nabla \phi, \nabla \psi)=\left(q_{\varepsilon}, \psi\right), \quad \forall \psi \in H_{0}^{1, *}(\Omega) .
$$

By Lax-Milgram theorem there is a unique solution, and $\phi \in H^{1, *}(\Omega) \cap H_{l o c}^{2}(\Omega)$.

Take a cut-off function $\zeta \in C_{0}^{\infty}\left(\mathbb{R}^{2}\right)$ such that $\zeta \equiv 1$ for $|x|<1, \zeta \equiv 0$ for $|x|>2$, and $0 \leq \zeta \leq 1$. Define $\zeta_{a}(x)=\zeta(x / a)$ and $\phi_{a}=\phi \zeta_{a}$. Let $q_{\varepsilon a}=-\Delta \phi_{a}$, then $q_{\varepsilon a} \in \mathcal{Q}$. When $a$ is large enough, we have $-\zeta_{a} \Delta \phi=-\Delta \phi=q_{\varepsilon}$, therefore for sufficiently large $a$, it holds that

$$
\begin{aligned}
\left\|q_{\varepsilon a}-q_{\varepsilon}\right\|_{\sharp} & =\left\|\Delta \phi_{a}-\Delta \phi\right\|_{\sharp} \\
& =\left\|\Delta \zeta_{a} \phi+2 \nabla \zeta_{a} \nabla \phi+\zeta_{a} \Delta \phi-\Delta \phi\right\|_{\sharp} \\
& =\left\|\Delta \zeta_{a} \phi+2 \nabla \zeta_{a} \nabla \phi\right\|_{\sharp} \\
& =\left(\left\|\Delta \zeta_{a} \phi+2 \nabla \zeta_{a} \nabla \phi\right\|_{0}^{2}+\left|\int_{\Omega}\left(\Delta \zeta_{a} \phi+2 \nabla \zeta_{a} \nabla \phi\right) \mathrm{d} x\right|^{2}\right)^{\frac{1}{2}} .
\end{aligned}
$$


Let us estimate the right hand side of (41). Since $|x|^{2} \ln ^{2}|x| \leq|x|^{3} \leq 8 a^{3}$ in the domain $D=\{x ; a \leq|x| \leq 2 a\}$, we have

$$
\begin{aligned}
\left\|\Delta \zeta_{a} \phi\right\|_{0}^{2} & \leq \frac{1}{a^{4}}\|\Delta \zeta\|_{0, \infty}^{2} \int_{D}|\phi(x)|^{2} \mathrm{~d} x \leq \frac{8}{a}\|\Delta \zeta\|_{0, \infty}^{2} \int_{D} \frac{|\phi(x)|^{2}}{|x|^{2} \ln ^{2}|x|} \mathrm{d} x \\
& \leq \frac{8 C}{a}\|\Delta \zeta\|_{0, \infty}^{2}\|\nabla \phi\|_{0, \Omega}^{2} \rightarrow 0, \quad(a \rightarrow \infty) .
\end{aligned}
$$

Analogously we have

$$
\left\|2 \nabla \zeta_{a} \nabla \phi\right\|_{0}^{2} \leq \frac{4}{a^{2}}\|\nabla \zeta\|_{0, \infty}^{2}\|\nabla \phi\|_{0, \Omega}^{2} \rightarrow 0 .
$$

For the second term of (41) we notice that

$$
-\int_{\Omega} \Delta \zeta_{a} \phi \mathrm{d} x=\int_{\Omega} \nabla \zeta_{a} \nabla \phi \mathrm{d} x
$$

therefore

$$
\left|\int_{\Omega}\left(\Delta \zeta_{a} \phi+2 \nabla \zeta_{a} \nabla \phi\right) \mathrm{d} x\right| \leq \int_{D}\left|\nabla \zeta_{a} \nabla \phi\right| \mathrm{d} x \leq \frac{1}{2}\|\nabla \zeta\|_{0, \Omega}\|\nabla \phi\|_{0, D} \leq C\|\nabla \phi\|_{0, D} \rightarrow 0 .
$$

To conclude there is a $a_{0}$ such that $\left\|q_{\varepsilon a}-q_{\varepsilon}\right\|_{\sharp}<\varepsilon$ for $a \geq a_{0}$. We fix such an $a_{\varepsilon}$.

Let $v_{q \varepsilon}=-\nabla \phi_{a_{\varepsilon}}$, then $v_{q \varepsilon} \in H_{0 c}(\Omega)$, and $\vec{\nabla} \cdot v_{q \varepsilon}=q_{\varepsilon a_{\varepsilon}}, \nabla \times v_{q \varepsilon}=0,\left\langle v_{q \varepsilon} \cdot \nu, 1\right\rangle=\int_{\Omega} q_{\varepsilon a_{\varepsilon}} \mathrm{d} x$. Consequently

$$
\left(\left\|\nabla \cdot v_{q \varepsilon}-q\right\|_{0}^{2}+\left|\left\langle v_{q \varepsilon} \cdot \nu, 1\right\rangle_{\partial \Omega}-\int_{\Omega} q \mathrm{~d} x\right|^{2}\right)^{\frac{1}{2}}=\left\|q_{\varepsilon a_{\varepsilon}}-q\right\|_{\sharp} \leq\left\|q_{\varepsilon}-q\right\|_{\sharp}+\left\|q_{\varepsilon a_{\varepsilon}}-q_{\varepsilon}\right\|_{\sharp}<2 \varepsilon .
$$

Therefore $v_{q \varepsilon}$ converges as $\varepsilon \rightarrow 0$. Let $v_{q}$ be the limit, then $v_{q}+V_{0}$ is the element in $W$ we are looking for.

Theorem 4.2. The problem $(39,40)$ admits a unique solution $(u, p)$, and $p=0$.

Proof. Because

$$
d(u, u)=\int_{\Omega}|\nabla \times u|^{2} \mathrm{~d} x+\int_{\Omega}|\nabla \cdot u|^{2} \mathrm{~d} x=\|u\|_{*}^{2},
$$

the bilinear form $d(\cdot, \cdot)$ is coercive. By Lemma 4.2

$$
\sup _{\substack{v \in W \\ v \neq 0}} \frac{b(v, q)}{\|v\|_{*}} \geq \frac{b\left(v_{q}, q\right)}{\left\|v_{q}\right\|_{*}}=\frac{\|q\|_{0}^{2}}{\|q\|_{\sharp}}=\|q\|_{\sharp} .
$$

Therefore the inf-sup condition holds, and there is a unique solution to the problem $(39,40)$.

Next let us show $p=0$. It is easy to see that $\nabla \cdot u \in Q$. Let $u_{n} \in V / V_{0}$, and $u_{n} \rightarrow u$, then $\int_{\Omega} \nabla \cdot u_{n} \mathrm{~d} x=$ $\left\langle u_{n} \cdot \nu, 1\right\rangle_{\partial \Omega}=0$, and $\int_{\Omega} \nabla \cdot u \mathrm{~d} x=0$ (in the generalized sense), so $\nabla \cdot u \in Q_{0}$. Let $q=\nabla \cdot u$ in (40), we will have $\nabla \cdot u=0$. According to Lemma 4.2 there is $v_{p}$ corresponding to the solution $p$. We take $v=v_{p}$ in (39) and obtain $(p, p)=0$, therefore $p=0$.

Remark 4.3. The solutions are not unique. They may differ from a function in $V_{0}$. It seems the most natural way to make the solution unique is to impose a boundary condition at the infinity, $\left.u\right|_{|x|=\infty}=0$, because a function in $V_{0}$ satisfying this condition is zero. Unfortunately we still can not prove the existence if this boundary condition is imposed. However we conjecture that existence holds under this condition, and we will see that in real computation, the lack of uniqueness is not a barrier. 


\section{INFINITE ELEMENT APPROXIMATION}

As a preparation for solving the exterior problems of the Darwin model, we recall the infinite element method for the exterior problems of the Stokes equation [9]. We use $P_{2}-P_{0}$ elements [3] as an example. For simplicity we assume that $\partial \Omega$ is a convex polygon. Denote $\Gamma_{0}=\partial \Omega$. We may assume the origin is in the interior of $\Gamma_{0}$. Taking a constant $\xi>1$, we draw the similar curves of $\Gamma_{0}$ with center $o$ and the constants of proportionality $\xi, \xi^{2}, \cdots, \xi^{k}, \cdots$, which are denoted by $\Gamma_{1}, \Gamma_{2}, \cdots, \Gamma_{k}, \cdots$ respectively. The domains between two polygons $\Gamma_{k-1}$ and $\Gamma_{k}$ are denoted by $\Omega_{k}$. Afterward each sub-domain is further divided into elements. We require that the triangulation of all $\Omega_{k}$ is geometrical similar to each other, and the triangulation of the entire domain $\Omega$ satisfies the condition of $C^{0}$ type, namely, the nodes of the mesh on $\Omega_{k}$ and the nodes of the mesh on $\Omega_{k+1}$ should coincide on $\Gamma_{k}$. Let $K$ be an element, then $u \in P_{2}(K)$ and $p \in P_{0}(K)$ on $K$. By this way we construct infinite element spaces, $S_{h}=\left\{u \in C(\bar{\Omega}) ;\left.u\right|_{K} \in P_{2}(K), \forall K\right\}$, and $M_{h}=\left\{p \in L^{2}(\Omega) ;\left.p\right|_{K} \in P_{0}(K), \forall K\right\}$. Let $g$ be the boundary value of $u$, then we take an approximation $g_{h}$, which is continuous, belongs to $P_{2}$ on each element edge, and $\int_{\Gamma_{0}} g_{h} \cdot \nu \mathrm{d} s=0$. The formulation of the infinite element approximation is: Find $u_{h} \in S_{h} \bigcap H^{1, *}(\Omega)$, $p_{h} \in M_{h}$, such that $\left.u_{h}\right|_{\Gamma_{0}}=g_{h}$, and

$$
\begin{aligned}
a\left(u_{h}, v\right)+b\left(v, p_{h}\right) & =(f, v), \quad \forall v \in S_{h} \cap H_{0}^{1, *}(\Omega), \\
b\left(u_{h}, q\right) & =0, \quad \forall q \in M_{h} .
\end{aligned}
$$

We have

Theorem 5.1. The problem (42, 43) admits a unique solution.

The proof of it is routine, thus omitted here.

Using the approach in [10] we can prove the following convergence theorem. For simplicity we assume that the boundary value is zero, $\left.u\right|_{\Gamma_{0}}=0$. We define the following weighted norms for $u$ and $p$.

$$
\begin{aligned}
|u|_{2(2)} & =\left\{\int_{\Omega} r^{2}\left|D^{2} u\right|^{2} \mathrm{~d} x\right\}^{\frac{1}{2}}, \\
|p|_{1(2)} & =\left\{\int_{\Omega} r^{2}|D p|^{2} \mathrm{~d} x\right\}^{\frac{1}{2}} .
\end{aligned}
$$

Theorem 5.2. We assume that the triangulation is regular, and

$$
\operatorname{meas} K \leq C_{0} h^{2} \operatorname{dist}(o, K)^{2},
$$

where $C_{0}$ is a positive constant, $h$ is the maximum diameter of the elements in $\Omega_{1}$, dist $(o, K)$ is the distance from $K$ to the origin o. If $|u|_{2(2)}$ and $|p|_{1(2)}$ are bounded, then

$$
\left|u-u_{h}\right|_{1} \leq C h\left\{|u|_{2(2)}+|p|_{1(2)}\right\}
$$

If $u \in H_{0}^{1, *}(\Omega)$, and $p \in L^{2}(\Omega)$, then

$$
\left|u-u_{h}\right|_{1} \rightarrow 0(h \rightarrow 0) .
$$

Proof. Using the inequalities in [10], it holds that

$$
\begin{gathered}
\inf _{w_{h} \in H_{0}^{1, *}(\Omega) \cap S_{h}}\left|u-w_{h}\right|_{1} \leq C h|u|_{2(2)}, \\
\inf _{q_{h} \in M_{h}}\left\|p-q_{h}\right\|_{0} \leq C h|p|_{1(2)} .
\end{gathered}
$$


Then $(44,45)$ follows from the standard result of the mixed finite element method [3].

We now turn to consider the infinite element method for the Darwin model. We need some auxiliary lemmas. We denote by $\xi^{k} \Omega$ the domain exterior to $\Gamma_{k}$, and consider a bounded domain $\Omega \backslash \xi^{k} \Omega$. The mesh restricted in this domain is also a mesh with finite number of elements. The finite element space defined on it is still denoted by $S_{h}$. Let

and let

$$
S_{h} \cap W\left(\Omega \backslash \xi^{k} \Omega\right)=\left\{\left.u \in S_{h}\right|_{\Omega \backslash \xi^{k} \Omega} ; u \times\left.\nu\right|_{\Gamma_{0}}=0, \int_{\Gamma_{0}} u \cdot \nu \mathrm{d} s=0\right\}
$$

$$
\|u\|_{*, \Omega \backslash \xi^{k} \Omega}=\left(\int_{\Omega \backslash \xi^{k} \Omega}|\nabla \cdot u|^{2} \mathrm{~d} x+\int_{\Omega \backslash \xi^{k} \Omega}|\nabla \times u|^{2} \mathrm{~d} x\right)^{\frac{1}{2}} .
$$

Lemma 5.1. $\|\cdot\|_{*, \Omega \backslash \xi^{k} \Omega}$ is a norm on $S_{h} \bigcap W\left(\Omega \backslash \xi^{k} \Omega\right)$.

Proof. If $\|u\|_{*, \Omega \backslash \xi^{k} \Omega}=0$, then $\Delta u=0$, so $u$ is a harmonic function. $u \in P_{2}$ on individual elements, hence $u \in P_{2}$ on $\Omega \backslash \xi^{k} \Omega$. We extend $u$ to the interior of $\Gamma_{0}$ analytically. Then we define the stream function $\psi$, such that $u=\left(\frac{\partial \psi}{\partial x_{2}},-\frac{\partial \psi}{\partial x_{1}}\right)$, then $\Delta \psi=0$. By the boundary condition of $u,\left.\frac{\partial \psi}{\partial \nu}\right|_{\Gamma_{0}}=0$, so $\psi$ is a constant in the interior of $\Gamma_{0}$. Therefore $u=0$.

Lemma 5.2. The space $W_{h}=\left\{u \in S_{h} ; \nabla \cdot u, \nabla \times u \in L^{2}(\Omega), u \times\left.\nu\right|_{\partial \Omega}=0,\langle u \cdot \nu, 1\rangle_{\partial \Omega}=0\right\}$ is a Hilbert space under the norm $\|\cdot\|_{*}$.

Proof. Because $V_{0} \cap S_{h}=\{0\},\|\cdot\|_{*}$ is a norm on $W_{h}$. Let us prove it is complete. Let $\left\{u_{n}\right\}$ be a Cauchy sequence with limit $u$. We are going to prove $u \in S_{h}$. $\left\{u_{n}\right\}$ is also a Cauchy sequence on $S_{h} \cap W\left(\Omega \backslash \xi^{k} \Omega\right)$. By Lemma 5.1, $u \in S_{h} \cap W\left(\Omega \backslash \xi^{k} \Omega\right)$. Since $k$ is arbitrary, $u \in S_{h}$.

Lemma 5.3. $W_{h} \subset H^{1, *}(\Omega)$.

Proof. Let $u \in W_{h}$. We define a cut-off function $\zeta \in C^{\infty}(\Omega)$, such that $\zeta \equiv 1$ near $\Gamma_{0}, \zeta \equiv 0$ near the infinity, and $0 \leq \zeta \leq 1$. Denote $\eta=1-\zeta$. We take an arbitrary $\varphi \in C_{0}^{\infty}(\Omega)$, then by the Green formula,

$$
\begin{aligned}
\int_{\Omega} \nabla(\varphi) \cdot \nabla(\eta u) \mathrm{d} x & =\int_{\Omega}(\nabla \cdot \varphi)(\nabla \cdot(\eta u)) \mathrm{d} x+\int_{\Omega}(\nabla \times \varphi) \cdot(\nabla \times(\eta u)) \mathrm{d} x \\
& \leq\|\varphi\|_{*}\|\eta u\|_{*}=|\varphi|_{1}\|\eta u\|_{*} .
\end{aligned}
$$

Since $u \in H^{1}$ on any bounded domain, $\|\eta u\|_{*}$ is bounded. Consequently $\eta u \in H_{0}^{1, *}(\Omega)$, that is, $u \in H^{1, *}\left(\xi^{k} \Omega\right)$ for large $k$. Then by $u \in H^{1}$ on any bounded domain we have $u \in H^{1, *}(\Omega)$.

Lemma 5.4. The norm $\|\cdot\|_{*}$ is equivalent to $\|\cdot\|_{1, *}$ on $W_{h}$.

Proof. It is the direct consequence of the closed graph theorem and Lemma 5.3.

We assume an inhomogeneous boundary condition, $u \times\left.\nu\right|_{\partial \Omega}=g$, and let $g_{h}$ be an approximation of $g$, then the formulation of the infinite element method is: Find $u_{h} \in S_{h} \cap H^{1, *}(\Omega), p_{h} \in M_{h}$, such that $u_{h} \times\left.\nu\right|_{\Gamma_{0}}=g_{h}$, $\int_{\Gamma_{0}} u_{h} \cdot \nu \mathrm{d} s=0$, and

$$
\begin{aligned}
d\left(u_{h}, v\right)+b\left(v, p_{h}\right) & =\int_{\Omega} B \cdot(\nabla \times v) \mathrm{d} x, \quad \forall v \in W_{h}, \\
b\left(u_{h}, q\right) & =0, \quad \forall q \in M_{h} .
\end{aligned}
$$

Theorem 5.3. The problem (46, 47) admits a unique solution. 
Proof. $d(\cdot, \cdot)$ is coercive. To verify the inf-sup condition we apply the inf-sup condition of the infinite element method for the Stokes equation,

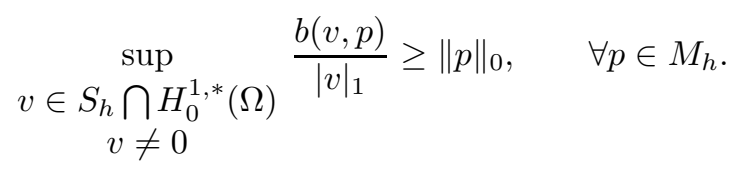

Now $W_{h} \supset S_{h} \cap H_{0}^{1, *}(\Omega)$, thus

$$
\sup _{\substack{v \in W_{h} \\ v \neq 0}} \frac{b(v, p)}{|v|_{1}} \geq\|p\|_{0}, \quad \forall p \in M_{h} .
$$

Moreover we have $\|v\|_{*}^{2} \leq 2|v|_{1}^{2}$, thus

$$
\sup _{\substack{v \in W_{h} \\ v \neq 0}} \frac{b(v, p)}{\|v\|_{*}} \geq \frac{1}{\sqrt{2}}\|p\|_{0}, \quad \forall p \in M_{h} .
$$

The relationship between the solutions of the infinite element method to the Stokes equation and to the Darwin model is the following:

Theorem 5.4. The solution to $(46,47)$ is a solution to (42, 43) with appropriate boundary value and inhomogeneous term.

Proof. We take $v$ in (46) such that $\left.v\right|_{\Gamma_{0}}=0$, then $v \in H_{0}^{1, *}(\Omega)$. Let a series $\left\{v_{n}\right\} \subset C_{0}^{\infty}(\Omega)$ tend to $v$ in $H_{0}^{1, *}(\Omega)$, then by the Green formula, $d\left(u_{h}, v_{n}\right)=a\left(u_{h}, v_{n}\right)$. Let $n \rightarrow \infty$ we verify that $u_{h}, p_{h}$ satisfy $(42,43)$.

Let us study convergence. For simplicity we assume $g=0$. We assume that the solution to $(39,40)$ satisfies $u \in H^{1, *}(\Omega)$. Then we can modify the formulation to: Find $u \in H^{1, *}(\Omega) \cap W, p \in L^{2}(\Omega)$, such that

$$
\begin{aligned}
d(u, v)+b(v, p) & =\int_{\Omega} B \cdot(\nabla \times v) \mathrm{d} x, \quad \forall v \in H^{1, *}(\Omega) \cap W, \\
b(u, q) & =0, \quad \forall q \in L^{2}(\Omega) .
\end{aligned}
$$

This is because the solution $u$ satisfies $\nabla \cdot u=0$, then $b(u, p)=0$ for all $p \in L^{2}(\Omega)$. Because $H^{1, *}(\Omega) \cap W$ is dense in $W$, uniqueness holds for the problem $(48,49)$.

We have the following result of error estimate:

Theorem 5.5. If $u$ and $p$ are solutions to $(48,49), u_{h}, p_{h}$ are solutions to $(46,47)$, in addition, $|u|_{2(2)}$ is bounded, then

$$
\left\|u-u_{h}\right\|_{*} \leq C h|u|_{2(2)} .
$$

Proof. This result follows from a theorem in [1]. Here we notice that the exact solution $p=0$, so $|p|_{1(2)}$ is bounded.

\section{Algorithm}

The algorithm for the Stokes equation is applied to the Darwin model, so we recall the algorithm of the infinite element method for the Stokes equation first.

The values of $u_{h}$ at the nodes on $\Gamma_{k}$ are arranged as a column vector, $y_{k}=\left(u_{1}^{(1)}, u_{2}^{(1)}, u_{1}^{(2)}, u_{2}^{(2)}, \cdots\right)^{T}$, where $u_{1}^{(j)}, u_{2}^{(j)}$ are the two components of $u_{h}$ at the $\mathrm{j}$-th node. The boundary value $g_{h}$ should satisfy $\int_{\Gamma_{0}} g_{h} \cdot \nu \mathrm{d} s=0$, 
that is, there is a vector $h$ such that $h^{T} y_{0}=0$. We take a particular function $q$ in the equation (43) as follows: $q=0$ on $\xi^{k} \Omega$, and $q=1$ on $\Omega \backslash \xi^{k} \Omega$, then we get

$$
0=\int_{\Omega \backslash \xi^{k} \Omega} \nabla \cdot u \mathrm{~d} x=\int_{\Gamma_{0}} u \cdot \nu \mathrm{d} s+\int_{\Gamma_{k}} u \cdot \nu \mathrm{d} s
$$

therefore $\int_{\Gamma_{k}} u \cdot \nu \mathrm{d} s=0$, namely, $h^{T} y_{k}=0$. We normalize $h$ to a unit vector, then construct an orthogonal matrix $T_{r}=[h, H]$, in which $h$ is the first column, and set $z_{k}=H^{T} y_{k}$, then there is a one to one correspondence between $z_{k}$ and $y_{k}$.

Given $y_{0}$ and $y_{1}$, we solve the Stokes equation on $\Omega_{1}$ by finite element method with boundary data $y_{0}, y_{1}$ on the given mesh. Let the approximate solution be $u_{h}$, then there are matrices $K_{0}, K_{0}^{\prime}$, and $A$, such that

$$
\int_{\Omega_{1}} \nabla u_{h} \cdot \nabla u_{h} \mathrm{~d} x=\left(\begin{array}{cc}
z_{0}^{T} & z_{1}^{T}
\end{array}\right)\left(\begin{array}{cc}
K_{0} & -A^{T} \\
-A & K_{0}^{\prime}
\end{array}\right)\left(\begin{array}{l}
z_{0} \\
z_{1}
\end{array}\right)
$$

The above expression is valid for all layers $\Omega_{k}$, hence the infinite element scheme is deduced to the following system of infinite equations:

$$
\begin{gathered}
-A z_{0}+K z_{1}-A^{T} z_{2}=0 \\
-A z_{1}+K z_{2}-A^{T} z_{3}=0 \\
\cdots \\
-A z_{k-1}+K z_{k}-A^{T} z_{k+1}=0
\end{gathered}
$$

where $K=K_{0}+K_{0}^{\prime}$. To solve this system directly is unnecessary. It is proved in [10] that there exists a real matrix $X$, called transfer matrix, such that $z_{k+1}=X z_{k}$, then from $z_{0}$ we can get the solution step by step. The approach to solve $X$ can be found in [10].

We need to introduce the definition of combined stiffness matrix $K_{z}$. Let $K_{z}=K_{0}-A^{T} X$, then it is proved in $[10]$ that

$$
\sum_{l=k+1}^{\infty} \frac{1}{2} \int_{\Omega_{l}}\left|\nabla u_{h}\right|^{2} \mathrm{~d} x=\frac{1}{2} z_{k}^{T} K_{z} z_{k}
$$

Being analogous to $(50)$ there are vectors $f_{k}, g_{k}$ for the inhomogeneous equation such that

$$
\frac{1}{2} \int_{\Omega_{k}} \nabla u_{h} \cdot \nabla u_{h} \mathrm{~d} x-\int_{\Omega_{k}} f \cdot u_{h} \mathrm{~d} x=\frac{1}{2}\left(z_{k-1}^{T} z_{k}^{T}\right)\left(\begin{array}{cc}
K_{0} & -A^{T} \\
-A & K_{0}^{\prime}
\end{array}\right)\left(\begin{array}{c}
z_{k-1} \\
z_{k}
\end{array}\right)+\left(\begin{array}{l}
z_{k-1}^{T} z_{k}^{T}
\end{array}\right)\left(\begin{array}{c}
f_{k} \\
g_{k}
\end{array}\right) .
$$

Let $\left\{z_{k}^{*}\right\}$ be a solution of the infinite element scheme to the equation, but it does not necessarily satisfy the boundary conditions. Let $\tilde{z}_{k}=z_{k}-z_{k}^{*}$, then $\tilde{z}_{k+1}=X \tilde{z}_{k}$. We have

$$
\begin{gathered}
z_{0}=\tilde{z}_{0}+z_{0}^{*} \\
z_{1}=\tilde{z}_{1}+z_{1}^{*}=X \tilde{z}_{0}+z_{1}^{*}=X\left(z_{0}-z_{0}^{*}\right)+z_{1}^{*} \equiv X z_{0}+q_{1} \\
\ldots \ldots \\
z_{k}=X z_{k-1}+q_{k}
\end{gathered}
$$

Let

$$
W_{k}=\sum_{l=k+1}^{\infty}\left\{\frac{1}{2} \int_{\Omega_{l}}\left|\nabla u_{h}\right|^{2} \mathrm{~d} x-\int_{\Omega_{l}} f \cdot u_{h} \mathrm{~d} x\right\}=\frac{1}{2} z_{k}^{T} K_{z} z_{k}+z_{k}^{T} h_{k}
$$


On the other hand

$$
W_{k}=\min _{z_{k+1}}\left\{\frac{1}{2}\left(\begin{array}{ll}
z_{k}^{T} & z_{k+1}^{T}
\end{array}\right)\left(\begin{array}{cc}
K_{0} & -A^{T} \\
-A & K_{0}^{\prime}
\end{array}\right)\left(\begin{array}{c}
z_{k} \\
z_{k+1}
\end{array}\right)+\left(\begin{array}{ll}
z_{k}^{T} & z_{k+1}^{T}
\end{array}\right)\left(\begin{array}{c}
f_{k+1} \\
g_{k+1}
\end{array}\right)+\frac{1}{2} z_{k+1}^{T} K_{z} z_{k+1}+z_{k+1}^{T} h_{k+1}\right\}
$$

We take the partial derivatives with respect to $z_{k+1}$, then let it be zero to obtain

$$
\left(K_{0}^{\prime}+K_{z}\right) z_{k+1}-A z_{k}+g_{k+1}+h_{k+1}=0
$$

We solve $z_{k+1}$ from this equation then substitute it into (53) and compare (53) with (52). It is deduced that

$$
h_{k}=A^{T}\left(K_{0}^{\prime}+K_{z}\right)^{-1}\left(g_{k+1}+h_{k+1}\right)+f_{k+1},
$$

and we have

$$
q_{k}=-\left(K_{0}^{\prime}+K_{z}\right)^{-1}\left(g_{k}+h_{k}\right) .
$$

Thus the procedure to solve inhomogeneous equations is:

(1) evaluate $h_{k}$ from the recurrence formula (54);

(2) evaluate $q_{k}$ from the formula (55);

(3) then get $z_{k}$.

Now let us go to the algorithm for the Darwin model. For the formulation $(46,47)$, we take a boundary value $\gamma_{h}$ for the Stokes equation such that $\gamma_{h} \times \nu=g_{h}$, and $\int_{\Gamma_{0}} \gamma_{h} \cdot \nu \mathrm{d} s=0$. Then solve the following problem: find $w_{h} \in S_{h} \cap H^{1, *}(\Omega), r_{h} \in M_{h}$, so that $\left.w_{h}\right|_{\Gamma_{0}}=\gamma_{h}$, and

$$
\begin{aligned}
a\left(w_{h}, v\right)+b\left(v, r_{h}\right) & =\int_{\Omega} B \cdot(\nabla \times v) \mathrm{d} x, \quad \forall v \in S_{h} \cap H_{0}^{1, *}(\Omega), \\
b\left(w_{h}, q\right) & =0, \quad \forall q \in M_{h} .
\end{aligned}
$$

The solutions to $(46,47)$ also satisfy

$$
\begin{aligned}
a\left(u_{h}, v\right)+b\left(v, p_{h}\right) & =\int_{\Omega} B \cdot(\nabla \times v) \mathrm{d} x, \quad \forall v \in S_{h} \cap H_{0}^{1, *}(\Omega), \\
b\left(u_{h}, q\right) & =0, \quad \forall q \in M_{h} .
\end{aligned}
$$

Let $u_{h}-w_{h}=U_{h}$ and $p_{h}-r_{h}=P_{h}$, then we have

$$
\begin{aligned}
a\left(U_{h}, v\right)+b\left(v, P_{h}\right) & =0, \quad \forall v \in S_{h} \cap H_{0}^{1, *}(\Omega), \\
b\left(U_{h}, q\right) & =0, \quad \forall q \in M_{h} .
\end{aligned}
$$

Let the vectors $y_{0}, y_{1}, \cdots, y_{k}, \cdots$ and $z_{0}, z_{1}, \cdots, z_{k}, \cdots$ correspond to $U_{h}$, then it holds that $z_{k+1}=X z_{k}$, which yields

$$
y_{k+1}=H X H^{T} y_{k}
$$

Let the function form of $(62)$ be $\left.U_{h}\right|_{\Gamma_{1}}=\left.Y U_{h}\right|_{\Gamma_{0}}$, where $Y$ is an operator. 
So far the problem $(46,47)$ is deduced to a finite element approximation on a bounded domain $\Omega_{1}$ for the following system of equations:

$$
\begin{aligned}
-\triangle u & =\nabla \times B, \quad x \in \Omega_{1}, \\
\nabla \cdot u & =0, \quad x \in \Omega_{1}, \\
u \times\left.\nu\right|_{\Gamma_{0}} & =g_{h}, \\
\int_{\Gamma_{0}} u \cdot \nu \mathrm{d} s & =0, \\
\left.\left(u-w_{h}\right)\right|_{\Gamma_{1}} & =\left.Y\left(u-w_{h}\right)\right|_{\Gamma_{0}},
\end{aligned}
$$

where $w_{h}$ is given. We solve the finite element problem on $\Omega_{1}$ with the same mesh, and get the solution $u_{h}$ to $(46,47)$ on $\Omega_{1}$. Using the formula $(62)$ and the solution $w_{h}$ to $(56,57)$ we are able to get the solution $u_{h}$ on the entire domain $\Omega$.

Using the matrix $X$ we can find $\lim _{|x| \rightarrow \infty} u_{h}$, which is given in [10].

\section{Numerical EXAMPleS}

We take a square with edge length 2 and consider the exterior domain. The original mesh is as following:

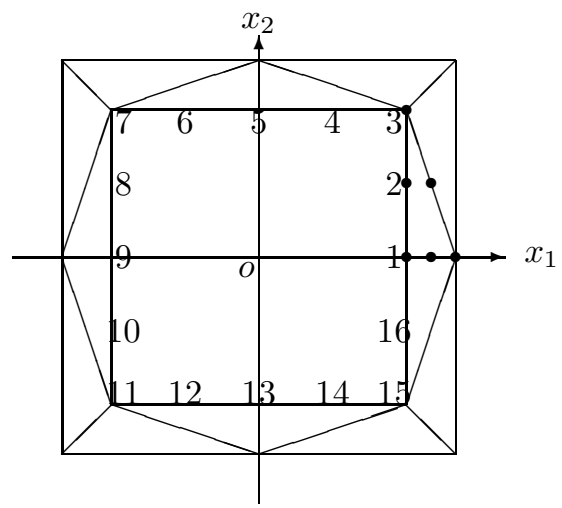

Example 1. $B=0$, and the exact solution is $u_{1} \equiv 0, u_{2} \equiv 1, p \equiv 0$. We take $\xi=1.2$, and use the value of the exact solution as the boundary value. And we get

$$
\|u\|_{2}=9.80 \times 10^{-13},\|p\|_{\infty}=3 \times 10^{-11}
$$

and

$$
\lim _{|x| \rightarrow \infty} u_{h}=\left(-7.06 \times 10^{-16}, 1\right) .
$$

The infinite element solution is actually the exact one for this example, so the error obtained here is just the round-off error. However this example hints that our method do work.

We have proved that the exact solutions are not unique. It is interesting to notice that the numerical solution obtained is just "the one we think about".

Example 2. $B=0$, and $u=\left(\frac{\cos 2 \theta}{r^{2}}, \frac{\sin 2 \theta}{r^{2}}\right)$. We use the original mesh which has the same structure as Example 1, and further we get mesh by refining one element into four small ones with the same size. The error is computed within the first $m+1$ layers. $N$ is the number of nodes on $\Gamma$. 
TABLE 1. Structured mesh, $\xi=1.1, m=20$.

\begin{tabular}{|c|c|c|c|c|c|c|}
\hline $\mathrm{N}$ & $\left\|u_{1}-u_{1 h}\right\|_{2}$ & Order & $\left\|u_{2}-u_{2 h}\right\|_{2}$ & Order & $\left\|u-u_{h}\right\|_{*}$ & Order \\
\hline 16 & 0.227782 & & 9.079201 & & 1.270610 & \\
\hline 32 & $5.689740 \mathrm{E}-02$ & 2.0012 & $1.288574 \mathrm{E}-02$ & 2.8168 & 0.764623 & 0.7327 \\
\hline 64 & $5.661458 \mathrm{E}-03$ & 3.3291 & $1.656519 \mathrm{E}-03$ & 2.9595 & 0.394076 & 0.9563 \\
\hline 128 & $6.330054 \mathrm{E}-04$ & 3.1609 & $1.964444 \mathrm{E}-04$ & 3.0760 & 0.197797 & 0.9945 \\
\hline
\end{tabular}

TABle 2. "Unstructured" Mesh, $\xi=1.1, m=20$.

\begin{tabular}{|c|c|c|c|c|c|c|}
\hline $\mathrm{N}$ & $\left\|u_{1}-u_{1 h}\right\|_{2}$ & Order & $\left\|u_{2}-u_{2 h}\right\|_{2}$ & Order & $\left\|u-u_{h}\right\|_{*}$ & Order \\
\hline 16 & 0.231510 & & $9.176199 \mathrm{E}-002$ & & 1.284699 & \\
\hline 32 & $5.668541 \mathrm{E}-002$ & 2.0300 & $1.309461 \mathrm{E}-002$ & 2.8089 & 0.764071 & 0.7497 \\
\hline 64 & $5.659193 \mathrm{E}-003$ & 3.3243 & $1.652787 \mathrm{E}-003$ & 2.9860 & 0.394033 & 0.9554 \\
\hline 128 & $6.821882 \mathrm{E}-004$ & 3.0524 & $2.121307 \mathrm{E}-004$ & 2.9619 & 0.198194 & 0.9914 \\
\hline
\end{tabular}

TABLE 3. Structured mesh, $\xi=1.2, m=20$.

\begin{tabular}{|c|c|c|c|c|c|c|}
\hline $\mathrm{N}$ & $\left\|u_{1}-u_{1 h}\right\|_{2}$ & Order & $\left\|u_{2}-u_{2 h}\right\|_{2}$ & Order & $\left\|u-u_{h}\right\|_{*}$ & Order \\
\hline 16 & $7.097883 \mathrm{E}-002$ & & $7.104025 \mathrm{E}-002$ & & 0.705941 & \\
\hline 32 & $9.723456 \mathrm{E}-003$ & 2.8678 & $9.723475 \mathrm{E}-003$ & 2.8691 & 0.385342 & 0.8734 \\
\hline 64 & $1.591538 \mathrm{E}-003$ & 2.6110 & $1.142021 \mathrm{E}-003$ & 3.0899 & 0.196713 & 0.9700 \\
\hline 128 & $1.341634 \mathrm{E}-004$ & 3.5684 & $1.491097 \mathrm{E}-004$ & 2.9371 & $9.862373 \mathrm{E}-002$ & 0.9961 \\
\hline
\end{tabular}

From Table 1, we can see first order convergence is obtained for $u$ under the norm $\|.\|_{*}$, which coincides with our theoretical result. And also for the function itself, we get third order convergence rate, which comes from the fact that $P^{2}$ element is used, and it hasn't been affected by using $P^{0}$ element for $p$.

Moreover, we change each mesh (for different $N$ ) above by disturbing each node (except those at corners) by the amount $(\Delta x, \Delta y)=\left(x_{*}, y_{*}\right) \times \frac{\min (h)}{5}$, where $\left(x_{*}, y_{*}\right)$ are identically independent random numbers from uniform $(-0.5,0.5)$. By this way, we obtain a set of "unstructured" meshes. Using these meshes, we get the same convergence rate, see Table 2.

Example 3. $B=0$, and $u=\left(\frac{\cos \theta}{r}, \frac{-\sin \theta}{r}\right)$. Structured mesh is used. Notice the solution in this example is less decreasing than $\frac{1}{r^{2}}$. Again we here see the similar convergence rate as in Example 2.

Example 4. Still we use the same solution as in Example 2, only here we focus on the effect of different parameter $\xi$. In Table 4, we list out the normal component of $u$ on $\Gamma_{0}$ when different $\xi$ is used. One can see the normal components at most points converge when $\xi$ decreases.

Then we use the same example as in Example 3 to see how the error changes with the parameter $\xi$. The error is computed in the domain $\left\{x ; 1 \leq\left|x_{1}\right|,\left|x_{2}\right| \leq 25\right\}$. In Table 5 , we can see with $\xi$ getting smaller, the error decreases, since we are using more elements to resolve our problem. However, when $\xi$ decreases to certain level, the error starts increasing. This is not very surprising because the shape of the element is becoming not 
TABLE 4. The normal component with different $\xi$.

\begin{tabular}{|c|c|c|c|c|c|c|c|}
\hline node & 1 & 2 & 3 & 4 & 6 & 7 & 8 \\
\hline$\xi=1.23$ & 1.0274 & 0.8269 & 0.4816 & 0.1898 & 0.6075 & 0.6539 & 0.4286 \\
\hline$\xi=1.17$ & 1.0273 & 0.8271 & 0.4841 & 0.1901 & 0.6073 & 0.6530 & 0.4296 \\
\hline$\xi=1.1$ & 1.0263 & 0.8276 & 0.4872 & 0.1902 & 0.6070 & 0.6511 & 0.4311 \\
\hline$\xi=1.07$ & 1.0255 & 0.8277 & 0.4885 & 0.1901 & 0.6070 & 0.6501 & 0.4319 \\
\hline$\xi=1.02$ & 1.0237 & 0.8278 & 0.4904 & 0.1897 & 0.6068 & 0.6481 & 0.4336 \\
\hline$\xi=1.005$ & 1.0231 & 0.8279 & 0.4912 & 0.1897 & 0.6065 & 0.6473 & 0.4338 \\
\hline exact & 1.0000 & 0.8304 & 0.4800 & 0.1792 & 0.6144 & 0.6400 & 0.4429 \\
\hline node & 9 & 10 & 11 & 12 & 14 & 15 & 16 \\
\hline$\xi=1.23$ & $1.45 \mathrm{E}-7$ & -0.4286 & -0.6539 & -0.6075 & 0.1898 & 0.4816 & 0.8269 \\
\hline$\xi=1.17$ & $-3.12 \mathrm{E}-8$ & -0.4296 & -0.6530 & -0.6073 & 0.1901 & 0.4841 & 0.8273 \\
\hline$\xi=1.1$ & $-8.29 \mathrm{E}-9$ & -0.4311 & -0.6511 & -0.6070 & 0.1902 & 0.4872 & 0.8276 \\
\hline$\xi=1.07$ & $1.58 \mathrm{E}-7$ & -0.4319 & -0.6501 & -0.6070 & 0.1901 & 0.4885 & 0.8277 \\
\hline$\xi=1.02$ & $2.32 \mathrm{E}-7$ & -0.4336 & -0.6481 & -0.6068 & 0.1897 & 0.4905 & 0.8278 \\
\hline$\xi=1.005$ & $-3.10 \mathrm{E}-4$ & -0.4344 & -0.6477 & -0.6068 & 0.1895 & 0.4909 & 0.8276 \\
\hline exact & 0.0000 & -0.4429 & -0.6400 & -0.6144 & 0.1792 & 0.4800 & 0.8304 \\
\hline node & 17 & 18 & 19 & 20 & 22 & 23 & 24 \\
\hline$\xi=1.23$ & 1.0274 & 0.8269 & 0.4816 & 0.1898 & 0.6075 & 0.6539 & 0.4286 \\
\hline$\xi=1.17$ & 1.0273 & 0.8273 & 0.4841 & 0.1901 & 0.6073 & 0.6530 & 0.4296 \\
\hline$\xi=1.1$ & 1.0263 & 0.8276 & 0.4872 & 0.1902 & 0.6070 & 0.6511 & 0.4311 \\
\hline$\xi=1.07$ & 1.0255 & 0.8277 & 0.4885 & 0.1901 & 0.6070 & 0.6501 & 0.4319 \\
\hline$\xi=1.02$ & 1.0237 & 0.8278 & 0.4905 & 0.1897 & 0.6068 & 0.6481 & 0.4336 \\
\hline$\xi=1.005$ & 1.0228 & 0.8277 & 0.4910 & 0.1897 & 0.6065 & 0.6474 & 0.4341 \\
\hline exact & 1.0000 & 0.8304 & 0.4800 & 0.1792 & 0.6144 & 0.6400 & 0.4429 \\
\hline node & 25 & 26 & 27 & 28 & 30 & 31 & 32 \\
\hline$\xi=1.23$ & $-1.48 \mathrm{E}-7$ & -0.4286 & -0.6539 & -0.6075 & 0.1898 & 0.4816 & 0.8269 \\
\hline$\xi=1.17$ & $2.98 \mathrm{E}-8$ & -0.4296 & -0.6530 & -0.6073 & 0.1901 & 0.4841 & 0.8273 \\
\hline$\xi=1.1$ & $-2.09 \mathrm{E}-8$ & -0.4311 & -0.6511 & -0.6070 & 0.1901 & 0.4872 & 0.8276 \\
\hline$\xi=1.07$ & $1.92 \mathrm{E}-7$ & -0.4319 & -0.6501 & -0.6070 & 0.1901 & 0.4885 & 0.8277 \\
\hline$\xi=1.02$ & $9.98 \mathrm{E}-7$ & -0.4336 & -0.6481 & -0.6068 & 0.1897 & 0.4905 & 0.8278 \\
\hline$\xi=1.005$ & $-4.87 \mathrm{E}-5$ & -0.4342 & -0.6475 & -0.6068 & 0.1895 & 0.4910 & 0.8278 \\
\hline exact & 0.0000 & -0.4429 & -0.6400 & -0.6144 & 0.1792 & 0.4800 & 0.8304 \\
\hline
\end{tabular}


TABLE 5. Errors with different $\xi$.

\begin{tabular}{|c|c|c|c|}
\hline$\xi$ & $\left\|u_{1}-u_{1 h}\right\|_{2}$ & $\left\|u_{2}-u_{2 h}\right\|_{2}$ & $\left\|u-u_{h}\right\|_{*}$ \\
\hline 5.0000 & 0.1113 & 0.1113 & 0.9160 \\
\hline 2.9240 & $3.7182 \mathrm{E}-002$ & $3.7182 \mathrm{E}-002$ & 0.5782 \\
\hline 2.2361 & $2.0647 \mathrm{E}-002$ & $2.0655 \mathrm{E}-002$ & 0.4676 \\
\hline 1.9037 & $1.4683 \mathrm{E}-002$ & $1.4685 \mathrm{E}-002$ & 0.4219 \\
\hline 1.7100 & $1.1997 \mathrm{E}-002$ & $1.1997 \mathrm{E}-002$ & 0.4005 \\
\hline 1.4300 & $9.5287 \mathrm{E}-003$ & $9.5286 \mathrm{E}-003$ & 0.3816 \\
\hline 1.3077 & $9.0638 \mathrm{E}-003$ & $9.0638 \mathrm{E}-003$ & 0.3805 \\
\hline 1.2085 & $8.9759 \mathrm{E}-003$ & $8.9723 \mathrm{E}-003$ & 0.3846 \\
\hline 1.1746 & $9.0021 \mathrm{E}-003$ & $8.9866 \mathrm{E}-003$ & 0.3871 \\
\hline 1.1502 & $9.0310 \mathrm{E}-003$ & $9.0237 \mathrm{E}-003$ & 0.3894 \\
\hline 1.1266 & $9.0783 \mathrm{E}-003$ & $9.0702 \mathrm{E}-003$ & 0.3920 \\
\hline
\end{tabular}

that "regular", then the condition number of the linear system we get is becoming worse. In application, one can reduce the parameter $\xi$ at the same time increasing the number of elements in each sub-domain $\Omega_{k}$.

Acknowledgements. We are grateful to the referee for calling our attention to the papers by Girault and Sequeira on exterior problem of Stokes equations.

\section{REFERENCES}

[1] P. Ciarlet Jr and J. Zou, Finite element convergence for the Darwin model to Maxwell's equations. Math. Modelling Numer. Anal. 31 (1997) 213-250.

[2] P. Degond and P.A. Raviart, An analysis of the Darwin model of approximation to Maxwell's equations. Forum Math. 4 (1992) 13-44.

[3] V. Girault and P.A. Raviart, Finite Element Methods for Navier-Stokes Equations. Springer, Berlin (1988).

[4] V. Girault and A. Sequeira, A well-posed problem for the exterior stokes equations in two and three dimensions. Arch. Ration. Mech. Anal. 114 (1991) 313-333.

[5] D.W. Hewett and C. Nielson, A multidimensional quasineutral plasma simulation model. J. Comput. Phys. 29 (1978) $219-236$.

[6] O.A. Ladyzhenskaya, The Mathematical Theory of Viscous Incompressible Flow. 2nd ed., Gordon and Breach, New York (1969).

[7] T.-T. Li and T. Qin, Physics and Partial Differential Equations. Higher Education Press, Beijing (1997).

[8] R. Temam, Navier-Stokes Equations, Theory and Numerical Analysis. 3rd ed., North-Holland (1984).

[9] L.-A. Ying, Infinite element approximation to axial symmetric Stokes flow. J. Comput. Math. 4 (1986) 111-120.

[10] L.-A.Ying, Infinite Element Methods. Peking University Press, Beijing and Vieweg and Sohn Verlagsgesellschaft mbH, Braunschweig/Wiesbaden (1995). 